NEW MATH FOR LIFE ACTUARIES

\title{
The fact
}

The model used in the technique of the Life Actuary is built on

i) probabilities of insured events, e.g. death, survival, disablement, just to mention the most common examples for such events,

ii) time value of money.

The model has been in use since more than 200 years (1762 Old Equitable) hence its practicability has been proved. Beyond that it has been the basis for insurance legislation all over the world. Hence the model is iron cast by law to be the right one.

\section{The challenge}

All models are wrong. Still we all know that they can nevertheless serve their purpose if they are applied with care. That means, if we add what the practitioners call "experience" and what the academics call "understanding of the limitations of the model" they become very valuable and useful tools. The question hence arises by how much a useful model may be wrong. My point is that for dealing with the time value of money (item ii) above the classical actuarial technique is nowadays so far off economic reality that it needs to be fundamentally revised. Here is a program for revision. The ideas are not new. One finds them in several scientific papers, some also published in the ASTIN Bulletin. My aim is to communicate these ideas more widely such that they will be applied and used.

1) Recognize that Life Assurance is always expressed in units. In private insurance (social insurance needs another definition of units) these units can always be thought of as units of a (possibly dynamic) portfolio. Such units can contain guarantees, one only needs to add the appropriate (European) options to the portfolio. As a special case, if the units are defined as the unit of the local currency, the portfolio is made up by Zero Coupon bonds plus options for the contractual guarantees.

2) Understand that in the language of units one unit always remains one unit. This has the great advantage that we can use the classical actuarial formulae at interest rate zero. This is the first discovery: The technical interest rate which good actuaries have always handled with suspicion has disappeared! The second discovery is even more convenient: The present value (in local currency) defined as the value of a corresponding (called replicating) portfolio can always (in principle) be read in todays newspaper! (at least if it has a reasonable financial section). 
To the non initiated the above may sound too simple and possibly more risky than how Life Assurance typically operates now. Let me address these two points.

\section{Simplicity}

In this new approach the actuary still needs to construct life tables, multiple decrement tables, probabilities for disablement etc. The only difference in his work is the fact that he can apply all his actuarial techniques calculating at interest rate zero. The more difficult part is the construction (in thought) of the portfolio which replicates the assured unit. Maybe some of us know already how to do that. If you don't, ask a Financial Engineer, but insist that he communicates to you exactly how this portfolio needs to be constructed and managed. He must in principle come up with the exact instructions how the Investment Department (if they wanted) could invest in the replicating portfolio.

Hence - in all fairness - the suggested new treatment of the time value of money is indeed conceptually simple to understand, but the practical implementation has its intricacies. So, nobody should fear to lose his or her job, if the new ideas should be applied in practice.

\section{Riskiness}

The financial risk (which is the most important for any Life Assurer) derives in the new approach from the fact that in reality Life Assurers often do not invest in the replicating portfolio. They may act so in the hope to make more profit by investing differently. If you replace "replicating portfolio" by the traditionally named "matching assets strategy", the same remark applies for the classical actuarial model. If however with the new approach the Life Insurer invests exactly in the replicating portfolio then the Financial Risk is Zero. The classical actuarial model - even under a matching assets strategy - can never achieve this since it does not account for contractual guarantees (most important in practice the guarantee of the technical interest rate). It is exactly this fact, which urges for a change in our cherished traditional actuarial model.

\section{How could the change happen?}

1) My appeal is first to my colleagues teaching actuarial science: Change the basis of your teaching along the principles outlined above. For the young generation of actuaries and financial engineers the new approach should become the obvious one. Therefore my title

NEW MATH

which could also be read

NUMAT

namely NUMeraire based

Actuarial

Teaching

$($ Numeraire $=$ Value of unit $)$ 
By the way, the time needed to explain the "numeraire" you can gain by omitting "compound interest".

2) To the actuarial consultants it should be obvious how to use the proposed approach for calculating Embedded Value. This would then also provide a common basis for such calculations hence lead to identical results - independent of who of us performs this calculation.

3) It is not my intention to make any proposals regarding legal and/or accounting rules. They rely on a process of consensus hence changes will always take time. Still from a risk management point of view one has to notice that the numeraire which has value 1 at time zero and value $(1+i)^{n}$ at each time $n$ is - theoretically not replicable.

HANS BÜHLMANN

PS My aim was to communicate ideas. Some fine points have been omitted on purpose - e.g. how to deal with surrender values. Such guarantees having the character of an American option can also be dealt with by the approach as outlined in my editorial, but need a technically different treatment than those guarantees which can be replicated by European options. 